\title{
Cefaléia Associada aos Ciclos Hormonais da Mulher
}

\author{
Rafaela Larsen Ribeiro* \\ Deusvenir de Souza Carvalho**
}

\section{RESUMO}

Os ciclos normais da vida da mulher estão associados a marcadores hormonais: menarca, ovulação, gravidez, amamentação, uso de contraceptivos, menopausa e terapia de reposição hormonal. Esses eventos e intervenções alteram os níveis e ciclos dos hormônios sexuais e podem causar mudança na prevalência ou intensidade das cefaléias. Cefaléias que acompanham a síndrome pré-menstrual parecem ser geradas centralmente, envolvendo o sistema nervoso central, incluindo os sistemas serotoninérgicos moduladores de dor. As medicações específicas para migrânea, como os triptanos, podem ser efetivas para o tratamento agudo da migrânea menstrual. Entretanto, é importante reconhecer as relações entre a migrânea e o ciclo menstrual, porque a cefaléla pode não responder aos tratamentos antimigranosos usuais.

\section{UNITERMOS}

Migrânea menstrual, fisiopatologia, tratamento.

Médica Pós-graduanda da Disciplina de Medicina Interna e Terapêutica da Escola Paulista de Medicina - Unifesp.

* Professor-adjunto e Chefe do Setor de Investigação e Tratamento das Cefaléias da Disciplina de Neurologia, Escola Paulista de Medicina - Unifesp.

\section{NTRODUÇÃO}

Migrânea é uma afecção freqüente que predomina no sexo feminino. Durante as diferentes fases do ciclo reprodutivo, a migrânea pode apresentar-se com características peculiares, tanto em suas manifestações clínicas e comorbidades como na resposta ao tratamento. Embora, de forma geral, a abordagem da crise migranosa seja a mesma da migrânea sem aura, a fisiologia das flutuações próprias do ciclo hormonal da mulher e das suas condições de gravidez e de amamentação justificam tratamentos específicos.

$\mathrm{O}$ uso de hormônios como contraceptivos pode influenciar a freqüência e a intensidade das crises migranosas, porém o tratamento da crise permanece inalterado. Atualmente, com progestinas de terceira geração, a piora da migrânea durante o uso de contraceptivos orais já não se apresenta como uma condição tão freqüente.

Estudos epidemiológicos indicam que a migrânea ocorre em $6 \%$ a $17 \%$ da população ${ }^{1}$. A prevalência da migrânea durante a infância nas meninas e nos meninos é semelhante, ocorrendo nítida preponderância no sexo feminino na puberdade, bem como nos adultos, numa razão de $2: 1$ a 4:12 . Na maioria das mulheres, foi observado que o início das crises de migrânea se dá no ano que antecede ou que sucede a menarca ${ }^{3,4}$. É fato bem observado que cefaléia e, particularmente, a migrânea se modificam nas diferentes fases do ciclo reprodutivo da mulher e podem ser reconhecidas: a migrânea exclusivamente menstrual, cujas crises ocorrem dois dias antes até o quarto ou o último dia do sangramento menstrual, prevalecendo em cerca de $10 \%$ a $20 \%$ e a migrânea associada à menstruação que prevalece em dois terços dos $\operatorname{casos}^{2,4}$.

Como a crise da migrânea, associada à menstruação ou exclusivamente menstrual, tem sido reportada como sendo mais intensa e menos responsiva ao tratamento que as outras migrâneas, as diversas abordagens para o tratamento desta incluem, além das opções de tratamento usadas para todas as migrâneas, como o tratamento agudo ou crônico, tratamentos específicos para melhor controle do ciclo menstrual e uma terapia hormonal adequada. 


\section{ASPECTOS FISIOPATOLÓGICOS}

Conhecida desde os tempos de Hipócrates, a migrânea menstrual era considerada como sintoma de manifestação do excesso de sangue que se agitava procurando uma forma de sair do corpo. Vista como uma doença menor e sem tratamento específico, durante séculos, a migrânea menstrual foi considerada uma situação relativamente normal pela qual algumas mulheres tinham que passar todos, ou quase todos, os meses.

Apenas ao final dos anos de $1940^{5}$ e no início dos anos de $1950^{6}$ foram propostos e avaliados tratamentos com progesterona, pois acreditava-se que o baixo nível plasmático deste hormônio fosse a causa da cefaléia. Surgem, assim, as teorias e os estudos sobre progesterona e migrânea menstrual, seguidos pelos estudos de outros hormônios, peptídeos e substâncias potencialmente envolvidas na patogênese da migrânea menstrual.

\section{Progesterona}

As falhas no tratamento da migrânea menstrual pela reposição de progestágenos foi interpretada por Dalton ${ }^{7}$ como próprias do hormônio sintético. Ao mesmo tempo em que baixam os níveis plasmáticos de progesterona endógena, os progestágenos não possuem a capacidade do hormônio natural de transporte de glucorticóides ou não ajudam na própria síntese de corticosteróides pelas glândulas supra-renais. O uso de progesterona por supositórios ou adesivos em muito melhorou a migrânea menstrual de pacientes tratadas por Dalton, enquanto o uso de progestágenos orais falhou nesse tratamento ${ }^{7}$.

\section{Estradiol}

Em 1972, Somerville ${ }^{8}$ conduziu um estudo clássico no qual um grupo de pacientes recebeu progestágeno e outro recebeu estradiol no período pré-menstrual. Todas as pacientes possuíam flutuações de níveis plasmáticos normais dos dois hormônios e sofriam de migrânea menstrual recorrente e bem definida. A manutenção artificial de níveis plasmáticos altos de progesterona não alterou o tempo para o aparecimento da cefaléia, embora tenha prolongado o tempo do sangramento uterino. A reposição de estradiol, ao contrário, não alterou o tempo do sangramento, mas prolongou o tempo do aparecimento da cefaléia em todas as pacientes em 3 a 9 dias. Apesar do pequeno número de pacientes tratadas, os resultados de Somerville são citados e respeitados até hoje.
Foi proposto que os altos níveis de estrógeno estimulariam os receptores $\alpha$-1-adrenérgicos da musculatura lisa pós-juncional vascular e os receptores $\alpha$-2-adrenérgicos que bloqueariam o acúmulo de noradrenalina na junção neurovascular ${ }^{9}$. Quando da queda de estrógeno, a normalização dessa situação seria inadequada em pacientes que sofrem de migrânea menstrual, resultando em alterações do fluxo sangüíneo regional e cefaléia ${ }^{9}$.

\section{Progesterona e estradiol}

Quando comparados os níveis e as flutuações plasmáticas de progesterona e estrógeno, em mulheres migranosas e não-migranosas, não se observam diferenças significativas. O nível plasmático de estrógeno eleva-se gradualmente até o momento da ovulação, quando, então, sofre rápida queda; segue-se nova elevação no período lúteo seguida por outra queda abrupta imediatamente antes do período menstrual. O nível plasmático de progesterona permanece baixo durante a menstruação e a fase folicular, elevando-se e atingindo um platô na fase lútea para cair rapidamente na fase pré-menstrual.

As crises de migrânea menstrual ocorrem tipicamente na fase em que os dois hormônios caem a seus níveis mais baixos. Além disso, poucas mulheres apresentam migrânea tanto em fase ovulatória quanto menstrual, quando o estrógeno se encontra em nível baixo, dificultando, assim, o estabelecimento de uma relação causa-conseqüência.

Parece mais provável, portanto, que o eixo hipotálamo-hipofisário seja responsável pelo desencadeamento de alterações que levam a crises de migrânea menstrual e que a causa dessas crises não seja simplesmente reflexo de uma variação nos níveis de estrógeno e/ou de progesterona plasmáticos. Em uma recente revisão ${ }^{10}$, observou-se que estudos placebo-controlados de tratamento hormonal com estrógenos, progestágenos ou progesterona mostraram resultados variáveis e conflitantes, tanto na síndrome pré-menstrual quanto na migrânea menstrual. Adicionalmente aos hormônios do ciclo menstrual, os opióides endógenos, a serotonina e o sistema adrenérgico central podem estar alterados na síndrome pré-menstrual ${ }^{11}$.

\section{Outros hormônios}

Agonistas do hormônio liberador de gonadotrofina (Gr RH-a) produzem um estado de hipogonadismo hipogonadotrófico por ação direta em receptores hipofisários de GnRH. Inibem ovulação e também os sintomas pré-menstruais, inclusive a cefaléia. No entanto, 
é um tratamento caro e envolve riscos de hipoestrogenismo, limitando sua avaliação e o papel do Gr RH na gênese e na manutenção da migrânea menstrual. Não parece ser uma disfunção primária deste hormônio a causa etiológica da migrânea ou da síndrome pré-menstrual. Assim como para outras substâncias endógenas, as alterações observadas parecem ser secundárias ao estado predisponente à síndrome pré-menstrual ${ }^{12}$.

\section{Prostaglandinas}

A produção de prostaglandinas (PG) pode estar particularmente aumentada nas mulheres que sofrem de migrânea menstrual. Conforme demonstrado por Carlson et $a l .{ }^{13}$, a injeção de PGE1 pode resultar em crises de migrânea. Por sua ação no útero, esta PG está aumentada no período menstrual normal e pode estar presente em níveis mais altos ou haver maior sensibilidade à PG nos casos de migrânea menstrual. Os níveis plasmáticos de PGE2 aumentam significativamente no período de migrânea menstrual ${ }^{14}$.

Níveis plasmáticos de 6-ceto-PGF1, metabólito estável da prostaciclina PGI2, encontram-se significativamente diminuídos nas pacientes com migrânea menstrual durante todo o ciclo ${ }^{15}$.

Corroborando a hipótese do envolvimento de PGs na migrânea menstrual, o tratamento com antiinflamatórios não hormonais no período pré-menstrual costuma resultar em melhora da cefaléia ${ }^{16}$.

\section{Serotonina}

No período perimenstrual, pacientes com migrânea menstrual apresentaram um acúmulo de serotonina em plaquetas ${ }^{17}$. As provas de agregação plaquetária foram semelhantes às dos controles, porém os níveis de 5HT foram muito superiores nas plaquetas das pacientes. Outro trabalho mostrou haver hiperagregabilidade plaquetária nas mulheres com migrânea menstrual no período prémenstrual, além de confirmar os níveis aumentados de 5HT nas plaquetas dessas pacientes ${ }^{18}$. Outros estudos mostraram redução da atividade serotoninérgica de plaquetas e baixos níveis plasmáticos de serotonina no período pré-menstrual de pacientes com síndrome prémenstrual ${ }^{19,20}$. Alguns autores sugerem que aberrações em níveis plasmáticos de serotonina nas cefaléias primárias, inclusive migrânea menstrual, sejam decorrentes de uma alteração serotoninérgica central ${ }^{21,22}$. Outros pesquisadores acreditam na disfunção da homeostase plaquetária ${ }^{23}$ e no aumento de catabolismo de $5 \mathrm{HT}^{24}$ no período pré-menstrual como causa da migrânea.

\section{Opióides endógenos}

Quando injetado na fase lútea, naloxona induz ao máximo o aumento de LH circulante em controles. Em pacientes com migrânea menstrual esta resposta está ausente 2 a 4 dias antes da menstruação, sugerindo falha da inibição opióide no hipotálamo ${ }^{25}$.

\section{Sistema adrenérgico}

Tanto a resposta da pressão arterial à tiramina ${ }^{26}$ quanto as flutuações de dopamina $\beta$-hidroxilase ${ }^{27}$, em pacientes com síndrome pré-menstrual, sugerem um sistema adrenérgico malfuncionante. Alterações funcionais de receptores $\alpha$-2-adrenérgicos também foram descritas em fases diferentes do ciclo menstrual ${ }^{28}$, inclusive com hipofunção desses receptores no período pré-menstrual $^{29}$. Bloqueadores dos receptores $\alpha$-2adrenérgicos alteram a resposta a opióides endógenos no nível central, sugerindo uma disfunção conjunta do sistema adrenérgico e endorfinérgico na síndrome prémenstrual ${ }^{11}$.

\section{Melatonina}

Pacientes que sofrem de migrânea menstrual apresentam menores níveis de melatonina em todas as fases do ciclo $^{30}$. Esse achado pode ser de relevância, considerando-se que a melatonina tem um papel no sistema antinociceptivo controlando a flutuação circadiana do limiar de dor e também inibe a síntese de PGE2.

\section{Prolactina}

Embora os níveis basais de prolactina sejam semelhantes em controles e em pacientes com migrânea menstrual, a liberação de prolactina como resposta ao hormônio estimulante da tiróide encontra-se significativamente aumentada nas pacientes ${ }^{31}$.

\section{TRATAME NTO}

\section{Migrânea menstrual}

A migrânea menstrual é definida com crises típicas de migrânea que ocorrem em todos, ou em quase todos, os meses, exclusivamente no período de dois dias que precedem a menstruação até o final do fluxo menstrual. 
Deve-se deixar claro que a maioria dos casos de migrânea pode apresentar piora das crises no período menstrual, sendo então denominada de "migrânea associada à menstruação". Podem coexistir tratamentos preventivos na migrânea menstrual, além de tratamento da crise.

Uma vez que a migrânea menstrual apresenta-se com previsão do período de crise e com aspectos fisiopatológicos próprios, surgem possibilidades de tratamentos específicos. Podem ser necessários diversos ciclos de tratamento para se obter o melhor resultado terapêutico e o diário de crises em muito ajuda na avaliação das respostas ao tratamento.

Os medicamentos utilizados na crise de migrânea menstrual bem caracterizada e com evidência de bons resultados $^{32}$ são o sumatriptano - $6 \mathrm{mg}$ subcutâneo (máximo de duas aplicações em 24 horas com mínimo de 6 horas entre as duas aplicações) ou o zolmitriptano $2,5 \mathrm{mg}$ via oral (máximo de 7,5 mg nas 24 horas). Também outros triptanos e os diversos tratamentos da migrânea sem aura podem ser utilizados.

No período perimenstrual é recomendado o uso de antiinflamatórios não esteróides (AINEs), como o naproxeno sódico, $550 \mathrm{mg}$ duas vezes por dia, iniciando dois dias antes da data prevista da menstruação, durante 5 a 7 dias. O uso de AINEs não exclui o emprego de triptanos para eventuais crises que ocorram durante o tratamento. A ergotamina, $1 \mathrm{mg}, 1 \mathrm{a} 2$ vezes ao dia por via oral, na fase perimenstrual, à semelhança dos AINEs, parece ser eficaz na prevenção da migrânea menstrual, porém exclui a possibilidade de usar triptanos caso haja uma crise. A associação de cafeína à ergotamina melhora a absorção e potencializa o efeito dessa droga, podendo ser utilizada por via oral.

O uso contínuo de sumatriptano, $25 \mathrm{mg}, 3$ vezes por dia, via oral, no período perimenstrual foi avaliado, em apenas um estudo aberto, com bom resultado.

$\mathrm{O}$ tratamento perimenstrual também pode ser feito com uso de estradiol percutâneo na dose de 1,5 mg de estradiol em gel, iniciando 2 dias antes da menstruação e mantendo por 7 dias. Um estudo aberto com uso de estradiol transdérmico, na dose de 100 microgramas a cada 2 dias, na fase perimenstrual, foi eficaz na prevenção, enquanto doses menores não mostraram eficácia ${ }^{33}$.

\section{Migrânea e gravidez}

Durante a gravidez existe a possibilidade de a migrânea piorar, melhorar, desaparecer ou até mesmo se manifestar. Essa característica é também observada em outras épocas do ciclo reprodutivo, possivelmente pela influência hormonal e pela regulação do eixo hipotálamo- hipofisário. A possibilidade de melhora da migrânea durante a gravidez, a partir do segundo trimestre, é cerca de $60 \%$, portanto, é possível que a maioria das pacientes não necessite do tratamento das crises.

Nas crises fracas são recomendadas medidas não medicamentosas como as utilizadas no tratamento da migrânea sem aura.

$\mathrm{O}$ tratamento medicamentoso fica restrito às drogas cujo benefício exceda os riscos para o feto. Deve-se ressaltar, no entanto, que, dada a falta de estudos que avaliem a eficácia de drogas no tratamento da crise migranosa na mulher grávida, não se conhece o risco e os benefícios de tais medicações para o feto.

Os analgésicos, como paracetamol e codeína, podem ser utilizados no início da crise migranosa. Alternativamente podem ser utilizados AINEs durante o primeiro e o segundo trimestres. Para crises fortes, clorpromazina, metoclopramida, dexametasona e meperidina podem ser utilizadas com risco mínimo. O uso de triptanos não foi devidamente avaliado e recomenda-se cautela. A ergotamina é contra-indicada.

\section{Migrânea e lactação}

Como nos casos de gravidez, o período de lactação exige restrição do uso de determinados medicamentos. Analgésicos comuns, como paracetamol e dipirona, são considerados como de risco mínimo, exceto o ácido acetilsalicílico que deve ser utilizado com cautela. Clorpromazina, haloperidol e metoclopramida não são recomendados. A ergotamina também é contra-indicada. Não há evidências suficientes com os triptanos.

\section{Migrânea, tensão pré-menstrual (TPM) e menopausa}

No tratamento das crises migranosas na menopausa, devem ser levados em conta os transtornos associados próprios dessa idade. Aproximadamente dois terços das mulheres melhoram da migrânea durante a menopausa fisiológica, enquanto outros dois terços pioram na menopausa cirúrgica.

O tratamento da migrânea durante a menopausa é o mesmo feito em outros períodos da vida.

Como a síndrome pré-menstrual divide muitas das características com os estados de depressão e de ansiedade, estes estão ligados à desregulação serotoninérgica, há evidências crescentes de que a serotonina possa também ser importante na patogênese da síndrome prémenstrual. Membros do grupo canadense de estudo colaborativo da síndrome pré-menstrual e fluoxetina ${ }^{34}$ 
mostraram que a fluoxetina, inibidora seletiva da recaptação de serotonina, é muito eficaz no tratamento da síndrome pré-menstrual (mais conhecida como TPM - tensão pré-menstrual). Os sintomas da síndrome prémenstrual aparecem regularmente durante a semana que precede e desaparecem após o sangramento menstrual. Tensão e irritabilidade estão entre os mais proeminentes sintomas que afetam cerca de $3 \%$ a $8 \%$ das mulheres norte-americanas no período reprodutivo. Neste estudo, duas doses de fluoxetina foram testadas, $20 \mathrm{mg}$ e $60 \mathrm{mg}$ por dia, sendo a dose de $20 \mathrm{mg}$ por dia de máxima eficácia terapêutica, reduzindo o potencial dos efeitos colaterais.

\section{Outros estudos}

Uma outra análise realizada pelo grupo de estudo do sumatriptano na migrânea menstrual ${ }^{35}$, representando a primeira investigação prospectiva, duplo-cega e placebocontrolada, sobre a eficácia e a tolerabilidade do sumatriptano no tratamento da migrânea menstrual, demonstrou que o sumatriptano, um agonista serotoninérgico do subtipo vascular do receptor, administrado subcutaneamente, é um efetivo e bem tolerado tratamento agudo para a migrânea menstrual.

A manipulação hormonal do ciclo menstrual com a terapia estrogênica tem obtido sucesso no tratamento das migrâneas menstruais. Murray \& Muse ${ }^{36}$ realizaram um estudo determinando que a terapia com a administração de um agonista da liberação de gonadotrofina (GnRH-a), sozinho ou seguido da terapia com estrógenoprogesterona constante, é um tratamento muito efetivo para pacientes cuidadosamente selecionadas com migrâneas perimenstruais intensas.

Em estudos duplo-cegos demonstrando que o tratamento agudo com sumatriptano efetivamente alivia a dor e os sintomas associados às migrâneas menstruais, soube-se também que a alta taxa do retorno das dores limita o uso deste tratamento para algumas pacientes. Assim, um estudo realizado por Newman et al..$^{37}$ demonstrou que a administração oral repetida do sumatriptano, mesmo na ausência da dor, caracterizando assim uma profilaxia, conseguiu efetivamente prevenir a migrânea perimenstrual, reduzindo dramaticamente a freqüência e a intensidade das crises de migrânea relacionadas à menstruação.

Grunfeld \& Gresty ${ }^{38}$ também realizaram um estudo muito interessante. Devido às similaridades entre os sintomas reportados pelos pacientes com migrânea e aqueles que tiveram a experiência de sentir "a doença do movimento" (motion sickness) levantou-se a questão se as duas condições envolveriam mecanismos comuns. Em mulheres, podendo a migrânea estar relacionada ao ciclo menstrual, foi sugerido que isso também poderia estar acontecendo com a "doença do movimento". Assim, o objetivo do estudo foi determinar se havia um modelo cíclico na ocorrência da migrânea e da "doença do movimento" entre membros da tripulação de uma corrida de iate em volta do mundo. Os participantes completavam questionários relatando sua suscetibilidade à "doença do movimento" e à migrânea e, adicionalmente, as mulheres detalhavam seu ciclo menstrual. Durante a corrida, os voluntários completavam um diário para recordar a ocorrência da "doença do movimento", migrânea, menstruação, medicação consumida e deveres a bordo do iate. As mulheres marinheiras estiveram mais propensas à "doença do movimento" do que os homens. Essa doença também estava ligada ao tempo despendido no mar. Os voluntários que sofreram de migrânea durante a corrida também foram mais susceptíveis à "doença do movimento". Como resultado, concluiu-se que existe um modelo distinto na ocorrência da "doença do movimento" e da migrânea relacionada ao ciclo menstrual.

Em outro estudo, Silberstein et al..$^{39}$ examinaram os benefícios da combinação entre o paracetamol, a aspirina e a cafeína para o tratamento da migrânea associada à menstruação comparado ao da migrânea não associada aos ciclos menstruais. Uma alta eficiência no tratamento da dor, da inaptidão e de sintomas associados foi detectada com o uso da combinação tanto em relação ao tratamento da migrânea associada à menstruação como ao da migrânea não associada aos ciclos menstruais.

\section{SUMMARY}

Menstrually related headache: mentrual migraine

The normal female life cycle is associated with a number of hormonal milestones: menarche, ovulation, pregnancy, breastfeeding, contraceptive use, menopause, and the use o replacement of sexual hormones. All these events and interventions promote modification of the levels and cycling of sexual hormones and may cause a change in the prevalence or intensity of headaches. Headaches occurring with premenstrual syndrome appear to be generated in the CNS neurons, including the serotoninergic pain modulating systems. Migraine-specific medications, such as the tryptans, may be effective for acute manegement of menstrual migraine. However, it is important to recognize the relationship between migraines and the menstrual cycle because these headaches may not be responsive to the usual antimigraine medications.

\section{KEYWORDS}

Menstrual migraine, pathophysiology, treatment.

\section{Referências}

1. Stewart WF, Lipton RB, Celentano DD, Reed ML. Prevalence of migraine headache in the United States. JAMA, 267:64-9, 1992.

2. Massiou H, Bousser MG. Influence of hormones on migrane. In: Olesen J, Tfelt-Hansen P, Welch KMA (eds.). The Headaches. Philadelphia, Lippincott Williams \& Wilkins, 2000, p. 261-7. 
3. Epstein MT, Hockaday JM, Hockaday TDR. Migraine and reproductive hormones throughout the menstrual cycle. Lancet, 1:543-8, 1975.

4. Macgregor A. Migraine in women. London, Martin Dunitz, 1999. p. 90

5. Singh I, Singh D. Progesterone in the treatment of migraine. Lancet, 1:745, 1947.

6. Greene R, Dalton KD. The premenstrual syndrome. Brit Med J, 1:1007, 1953.

7. Dalton KD. Progesterone suppositories and pessaries in the treatment of menstrual migraine. Headache, 12:150-8, 1973.

8. Somerville BW. The role of oestradiol withdrawal in the etiology of menstrual migraine. Neurology, 22:355-65, 1972.

9. Welch KMA, Darnely D, Simkings RT. The role of estrogen in migraine: a review and a hypothesis. Cephalalgia, 4:22736, 1984.

10. DiCarlo C, Bifulco G, Pellicano M, Napolitano V, Nappi C. Hormonal treatment of premenstrual syndrome. Cephalalgia, 17(suppl. 20):17-9, 1997.

11. Fioroni L, Martignoni E, Facchinetti F. Changes of neuroendocrine axes in patients with menstrual migraine. Cephalalgia, 15:297-300, 1995

12. Benedetto C, Allais G, Ciochetto D, DeLorenzo C. Pathopysiological aspects of menstrual migraine. Cephalalgia, 17(suppl. 20):2-4, 1997.

13. Carlson LA, Ekelund LG, Oro L. Clinical and metabolic effects of different doses of prostaglandin $\mathrm{E}$ in man. Acta Med Scand, 183:423-30, 1968

14. Nattero G, Allais G, DeLorenzo C, Torre E, Ancona M, Benedetto $C$, Massobrio M. Menstrual migraine: new biochemical and psychological aspects. Headache, 28:1037, 1988.

15. Nattero G, Allais G, DeLorenzo C, Benedetto C, Zonca M, Melzi E, Massobrio M. Relevance of prostaglandins in true menstrual migraine. Headache, 29:233-8, 1989.

16. Sances G, Martignoni E, Fioroni L, Blandini F, Facchinetti F, Nappi G. Naproxen sodium in menstrual migraine prophylaxis: a double-blind placebo controlled study. Headache, 30:7059, 1990

17. Benedetto $\mathrm{C}$, Massobrio $\mathrm{M}$, Zonca $\mathrm{M}$. Menstrual migraine: a possible pathogenic implication of platelet function. Gynecol Endocrinol, 1:345-53, 1987.

18. Leira R, Martinez F, Castillo J, Rodriguez JR, Lema M, Noya $M$. Hormonal profile and changes in platelet aggregation in menstrual migraine. Neurologia, 5:306-9, 1990.

19. Taylor DL, Mathew RJ, Ho BT, Weinamn ML. Serotonin levels and platelet uptake during prementrual tension. Neuropsychobiology, 12:16-8, 1984.

20. Rapkin AJ, Edelmuth E, Chang LC, Reading AE, McGuire MT, Su TP. Whole blood serotonin in premenstrual syndrome. Obstet Gynecol, 70:533-7, 1987.

21. Silberstein SD, Merriam GR. Estrogens, progestins and headache. Neurology, 41:786-93, 1991.

22. D'Andrea G, Hasselmark L, Cananzi AR, Alecci M, Perini F, Zamberlan F, Welch KMA. Metabolism and menstrual cycle rhytmicity of serotonin in primary headaches. Headache, 35:216-21, 1995.

23. Allais G, Facco G, Ciochetto D, DeLorenzo C, Piore M, Benedetto C. Patterns of platelet aggregation in menstrual migraine. Cephalalgia, 17(suppl. 20):39-41, 1997.

24. Fioroni L, D'Andrea G, Alecci M, Cananzi A, Facchinetti F. Platelet serotonin pathway in menstrual migraine. Cephalalgia, 16:427-30, 1996.
25. Facchinetti F, Nappi G, Petraglia F, Volpe A, Genazzani AR. Oestradiol/progesterone inbalance and the premenstrual syndrome. Lancet 1983; ii:1302.

26. Ghose K, turner P. The menstrual cycle and tyramine pressor response test. Br J Clin Pharmacol, 4:500-2, 1977.

27. Magos AL, Brincat M, Zikha EJ, Studd JWW. Serum dopamineb-hydroxilase activity in mesntraul migraine. J Neurol Neurosurg Psychiatry, 48:328-31, 1985.

28. Jones SB, Bylund DB, Rieser CA, Shekim WO, Byer JA, Carr GW. $\alpha$-2-adrenergic receptor binding in human platelets: alterations during the menstrual cycle. Clin Pharmacol Ther, 34:90-6, 1983.

29. Facchinetti F, Martignoni E, Nappi G, Fioroni L, Sances G, Genazzani AR. Premenstrual failure of $\alpha$-adrenergic stimulation on hypothalamus-pituitary responses in menstrual migraine. Psychosom Med, 51:550-8, 1969.

30. Murialdo G, Fonzi S, Costelli P, Solinas GP, Parodi C, Marabini $S$. Urinary melatonin excretion though out the ovarian cycle in menstrual related migrane. Cephalalgia, 14:205-9, 1994.

31. Awaki E, Takeshima T, Takahashi K. A neuroendocrinological study in female migraneurs: prolactin and thyroid stimulting hormone responses. Cephalalgia, 9:187-93, 1989.

32. Consenso da Sociedade Brasileira de Cefaléia. Recomendações para o tratamento da crise migranosa. Arq Neuropsiquiatr, 58(2-A):371-89, 2000.

33. Pfaffenrath, V. Efficacy and safety of percutaneous estradiol vs placebo in menstrual migrane. Cephalalgia, 13 (suppl. 13):244, 1993.

34. Steiner $M$, Steinberg $S$, Stewart $D$ et al. Fluoxetin in the treatment of premenstrual dysphoria. N Engl J Med, 332:1529-34, 1995.

35. Facchinetti F, Bonellie G, Kangasniemi P, Pascual J, Shuaib A. The efficacy and safety of subcutaneous sumatriptan in the acute treatment of menstrual migrane. Obstet Gynecol, 86:911-6, 1995.

36. Murray SC, Muse KN. Effective treatment of severe menstrual migraine headaches with gonadotropin-realising hormone agonist and "add-back" therapy. Fertil Steril, 67(2):390-3, 1997.

37. Newman LC, Lipton RB, Lay CL, Solomon S. A pilot study of oral sumatriptan as intermitent prophylaxis of menstruationrelated migrane. Neurology, 51:307-9, 1998.

38. Grunfeld E, Gresty MA. Relationship between motion sickness, migrane and menstruation in crew members of a "round the world" yacht race. Brain Research Bull, 47(5):433-6, 1998.

39. Silberstein SD, Armellino JJ, Hoffman HD, Battikha JP, Hamelsky SW, Stewart WF, Lipton RB. Treatment of menstruation-associated migraine with the nonprescription combination of acetaminophen, aspirin, and caffeine: results from three randomized, placebo-controlled studies. Clin Ther, $21(3): 475-91,1999$

\section{Endereço para correspondência:}

Deusvenir de Souza Carvalho

Disciplina de Neurologia

Rua Pedro de Toledo, 650

Ed. Jairo Ramos $-2^{\circ}$ andar, sala 48

CEP 04039-002 - São Paulo, SP

E-mail: deusveni@provida.org.br 